\title{
Prospects for Higgs and BSM Searches at LHC
}

\author{
Daniela Rebuzzi ${ }^{1 *}$ \\ 1- Pavia University and INFN, Sezione di Pavia \\ Via A. Bassi, 6 - 27100 Pavia, Italy
}

\begin{abstract}
The Large Hadron Collider is undergoing its final installation at CERN. The first $p p$ collisions at $14 \mathrm{TeV}$ in the center of mass are foreseen by summer 2008. ATLAS and CMS commissioning is well underway, the two experiment installations are concluding. Despite the initial phase will be devoted to the understanding and the calibration of the detectors and to the re-discovery of the Standard Model, discoveries in the Higgs and beyond the Standard Model physics can be achieved in the first years.

The present paper presents, with increasing integrated luminosity order, the ATLAS and CMS discovery reaches of the LHC first data taking period.
\end{abstract}

\section{Introduction}

The Standard Model (SM) has been proved by the LEP experiments with precisions up to $0.1 \%$. Nevertheless there are issues connected with it which are still open. The most crucial is related to how fermions and vector bosons acquire their mass after the Electroweak Symmetry breaking (EWSB). The SM cannot exist without a neutral scalar particle, the Higgs boson. If an Higgs particle exists, the problem of the quadratically divergent corrections to its mass has to find a solution.

In the SM, a fine tuning cares of them, but a more elegant solution would be preferred, like the ones predicted by several Beyond the Standard Model (BSM) models. If no Higgs particles is found, alternative solutions should be invoked to explain the EWSB, like Technicolor or Compositeness.

The Large Hadron Collider (LHC), currently in its final installation stage at CERN, has the unique possibility to explore the TeV energy scale and to search for new physics and new particles with mass up to $5 \mathrm{TeV}$. The first $p p$ collision, foreseen by the end of 2007 , will have a center of mass energy of $900 \mathrm{GeV}\left(\mathcal{L}=10^{29} \mathrm{~cm}^{-2} \mathrm{~s}^{-1}\right)$. The first interactions at 14 $\mathrm{TeV}$ are scheduled for summer 2008 , then one can estimate to collect (per experiment) up to $1 \mathrm{fb}^{-1}$ by the end of $2008\left(\mathcal{L}<10^{33} \mathrm{~cm}^{-2} \mathrm{~s}^{-1}\right)$ and up to $10 \mathrm{fb}^{-1}$ by the end of $2009(\mathcal{L}$ $\left.=10^{33} \mathrm{~cm}^{-2} \mathrm{~s}^{-1}\right)$.

The present paper discusses how the discovery possibilities of ATLAS and CMS, the two general purpose experiments which will take data at the LHC, could evolve together with the collected integrated luminosity $L$ and progressive knowledge of the detector performance. The achievements on Higgs and BSM physics will be discussed, starting from $100 \mathrm{pb}^{-1}$ to $5 \mathrm{fb}^{-1}$, with focus on processes with large cross section and small SM backgrounds and possibly with clear kinematic signatures (peak, edge) and leptonic final states.

\section{High mass di-lepton resonances $\left(100 \mathrm{pb}^{-1}\right)$}

Resonances in lepton-lepton invariant mass distributions are predicted by several BSM models. The di-lepton channel offers a prominent and clear signature, with two electrons or two

*email address: daniela.rebuzzi@pv.infn.it 
muons in the finale state, and can be considered a benchmark for the theory and the detectors.

The background is dominated by the Drell-Yan production; other backgrounds $(Z Z$, $Z W, W W, t \bar{t})$ are smaller and easily reducible thorough kinematic selections. The main selection cut requires a pair of high $p_{T}$ isolated opposite-sign leptons. The favorite discovery channel is the di-electron, since the calorimeters have an good resolution (lower that 1\%) in the $\mathrm{TeV}$ region. $Z^{\prime} \rightarrow \mu \mu$ is anyway possible, also with the imperfect knowledge of the muon systems that ATLAS and CMS will have at the early stage of data taking.

A $1 \mathrm{TeV}$-reach is achievable for all the models, already with $100 \mathrm{pb}^{-1}$. A signal can be reach very early, within the first weeks of data taking at $14 \mathrm{TeV}$ energy.

\section{Micro Black Holes $\left(100 \mathrm{pb}^{-1}\right)$}

An integrated luminosity of $100 \mathrm{pb}^{-1}$ would be enough to provide a first evidence of high- $p_{T}$ objects as the micro black holes (BH), foreseen in large Extra-Dimension theories. Microscopic black holes can be produced when $E_{c m}>M_{P l}$ (which is at TeV scale in these models) and two colliding partons have impact parameter smaller that the radius of a $\mathrm{BH}$.

The partonic cross section estimation at LHC is motivated by (semi)classical geometric arguments and is of the order of $100 \mathrm{pb}$, potentially large. At high luminosity more than one $\mathrm{BH}$ with mass larger than $5 \mathrm{TeV}$ can be created per second at LHC. The BHs behave roughly as black bodies and evaporate in a short time $\left(10^{-27} \mathrm{~s}\right)$ via Hawking radiation, giving rise to a large multiplicity of SM particles in the final state (including gauge bosons and Higgs bosons), isotropically distributed. The separation from the background, which mostly consists of QCD jets, can occur by means of the event shape variables.

The $5 \sigma$ discovery contours shows that the first collected $100 \mathrm{pb}^{-1}$ would allow to explore the $\mathrm{BH}$ mass region up to $4 \mathrm{TeV}$, for $n<7$.

\section{SUSY early searches $\left(1 \mathrm{fb}^{-1}\right)$}

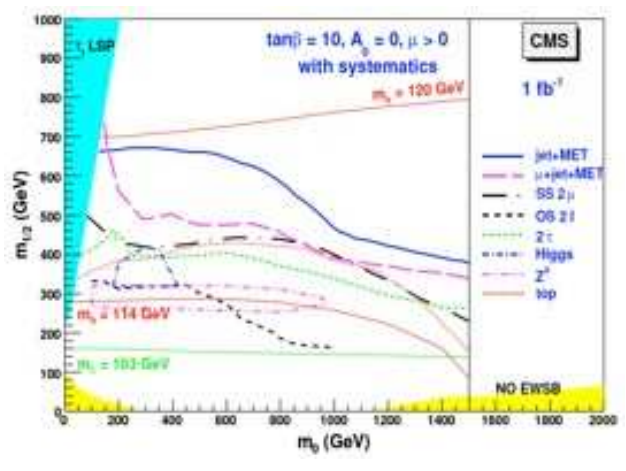

Figure 1: CMS $5 \sigma$ discovery reaches with $L=1 \mathrm{fb}^{-1}$ for different SUSY signatures.

in pairs and the lightest supersymmetric particle (LSP) is stable. Therefore, sparticles decay in lighter sparticles or in SM particles, and the decay chain eventually ends up in the LSP.
Supersymmetry (SUSY) is the most popular template for the exploration of new physics at the LHC. In SUSY for each fermionic state there is a bosonic partner with the same mass and the same couplings, therefore SUSY is a broken symmetry. There are several mechanisms to mediate SUSY breaking, the main being the gravity mediation (mSUGRA), the gauge mediation, the anomaly mediation.

The classical SUSY signatures are based on the so-called Minimal Supersymmetry Model (MSSM), which has only soft SUSY breaking terms and a minimal particle content. The additional R-parity conservation, which is imposed, has two consequences which crucially affect the phenomenology: the superparticles are produced 
A typical signature for a SUSY event at the LHC consists of jets + leptons + large missing $E_{T}$, from the LSP which escapes the detection.

LHC can cover a wide range of parameters of the MSSM, providing a crucial test for the MSSM and low-energy SUSY. Figure 1 shows the $5 \sigma$ discovery reaches for CMS, for different signatures, in the mSUGRA parameter space $\left(m_{0}=\right.$ universal scalar mass, $m_{1 / 2}=$ universal gaugino mass) and for a given choice of the model parameters.

The cross sections are high (1-100 pb for squark/gluino masses $\sim 0.5-1.0 \mathrm{TeV}$ ), therefore the statistic will not be a limit to the SUSY discovery at LHC: $100 \mathrm{pb}^{-1}$ will allow to explore the mass range up to $1.3 \mathrm{TeV}, 1 \mathrm{fb}^{-1}$ will increase this limit up to $1.8 \mathrm{TeV}$ and 10 $\mathrm{fb}^{-1}$ up to $2.2 \mathrm{TeV}$. The discovery is bound by the time needed to understand the detector performance ( $E_{T}$ miss tails, lepton identification, etc.) and to collect sufficient statistics of SM control samples. Indeed, SUSY will manifest itself as an excess over the SM background, for instance in the effective mass $\left(M_{\text {eff }}=\sum_{i=1}^{4}\left|p_{T}\left(j_{e} t_{i}\right)\right|+E_{T}^{m i s s}\right)$ distribution. More time will be needed to understand the backgrounds (SM and instrumental) and therefore before convincing than a SUSY excess can be claimed.

\section{$5 \quad$ SM Higgs Discovery Potential}

As outlined above, if the Higgs mechanism describes the EWSB, a neutral scalar particle, the Higgs boson, must exist. One of the main goals of LHC is to explore the existence of an Higgs particle with mass below $1 \mathrm{TeV}$. The current limits on the Higgs mass come from the LEP direct search $\left(M_{H}>114.4 \mathrm{GeV}\right.$ at $\left.95 \% \mathrm{CL}\right)$ and from the fit to the EW data $\left(M_{H}<\right.$ $144 \mathrm{GeV}$ at $95 \% \mathrm{CL}$, January 2007). If one wants to investigate on an early discovery or evidence of a SM Higgs boson, channels with an high branching ratio and not overwhelmed by SM backgrounds should be considered. Good candidates for a first observation are the $H \rightarrow W W^{(*)}$ and the $H \rightarrow Z Z^{(*)}$ channels (with the Higgs produced both via ggF and $\mathrm{VBF}$ ), due to the high production rate, the branching ratio above $90 \%$, the good trigger possibility and the signal/background ratio.

\section{$\mathbf{5 . 1} \quad H \rightarrow W W^{(*)} \rightarrow l l \nu \nu\left(\mathbf{1}-\mathbf{3} \mathrm{fb}^{-1}\right)$}

This channel has two leptons and two neutrinos in the final state. Due to the neutrinos, which escape the detection, the Higgs mass peak cannot be reconstructed. The main backgrounds are due to $t \bar{t}$ and the continuum $W W$ production. The analysis selection requires two oppositely charged isolated and high- $p_{T}$ leptons and large $E_{T}^{m i s s}$ (from neutrinos). Contrary to the background, the leptons from the signal are spin correlated, and the request of a small opening angle is the most effective for the background reduction. A reduction of the top background can be obtained also by vetoing events with jet activity.

A $5 \sigma$ Higgs evidence of a SM Higgs is possible already with $1 \mathrm{fb}^{-1}$ if the particle is in the $160-168 \mathrm{GeV}$ mass range, which increase to $155-175 \mathrm{GeV}$ with $3 \mathrm{fb}^{-1}$. Therefore a $5 \sigma$ Higgs signal might be observable in the first year at LHC. Actually, being $H \rightarrow W W^{(*)}$ a counting channel (no mass peak), confirmation from other channels will be needed before claiming the discovery. As for SUSY, the understanding of the SM background from data is of crucial importance. 


$$
\mathbf{5 . 2} \quad H \rightarrow Z Z^{(*)} \rightarrow 4 l\left(\mathbf{3}-\mathbf{4} \mathrm{fb}^{-1}\right)
$$

The combination of a narrow reconstructed mass peak and relatively low backgrounds makes this channel the golden one for the SM Higgs discovery, above $180 \mathrm{GeV}$ when the cross section for two $Z$ on-mass-shell opens. The main backgrounds are $Z b \bar{b}$ and $t \bar{t}$ (reducible) and the irreducible $Z Z$ continuum production.

The analysis selection requires isolated high- $p_{T}$ leptons (muons, electrons) from the interaction vertex. The significance can be improved by applying $Z$ mass constraint and by cutting events out of a window around the Higgs mass peak. Crucial for this channel are the efficiency and the resolution on the lepton reconstruction and a good impact parameter resolution to suppress $Z b \bar{b}$ and $t \bar{t}$ backgrounds.

The region around $M_{H} \sim 200 \mathrm{GeV}$ can be investigated with $3 \mathrm{fb}^{-1}$, while $5 \mathrm{fb}^{-1}$ will allow for a $5 \sigma$ discovery on the large mass range, from 200 to $450 \mathrm{GeV}$.

\section{Dynamical EWSB: Technicolor $\left(5 \mathrm{fb}^{-1}\right)$}

Technicolor provides an alternative to the Higgs mechanism. It introduces a new strong interaction providing a dynamical nature for EWSB. The new QCD-like force acts on particles named technifermions at a scale $\Lambda_{T C} \sim \nu_{\text {weak }}=246 \mathrm{GeV}$. In this picture there is no need for Higgs boson(s) and the fine tuning problem is automatically removed.

A good candidate for an early discovery is the $\rho_{T C} \rightarrow W Z$ channel, which has the advantages of a clean leptonic final state with isolated leptons and the possibility to apply $W$ and $Z$ the kinematic constraint. The background is dominated by the SM processes $W Z, Z Z, Z b \bar{b}$ and $t \bar{t}$. The $5 \sigma$ discovery contours in the $\left[M\left(\rho_{T C}\right), M\left(\pi_{T C}\right)\right]$ parameter space shows a signal sensitivity starting from $3 \mathrm{fb}^{-1}$.

\section{Conclusions}

The first LHC physics run at $14 \mathrm{TeV}$ are foreseen in summer 2008. The initial emphasis will be focused on understanding the detector performance and SM processes but both ATLAS and CMS will have the possibility to achieve important discoveries in the SM Higgs sector and for the BSM physics.

\section{Acknowledgments}

I would like to express my gratitude to: G. Polesello, F. Gianotti, M. Schumacher, V. Vercesi, L. Fayard, S. Eno, A. Nikitenko, A. Nisati, S. Rosati.

\section{References}

[1] Slides:

http://indico. cern. ch/contributionDisplay . py? contribId=121\&; sessionId=9\&; conf Id=9499

[2] CMS Physics TDR, Volume II, CERN-LHCC-2006-021.

[3] ATLAS TDR, Volume II, CERN/LHCC/99-15. 